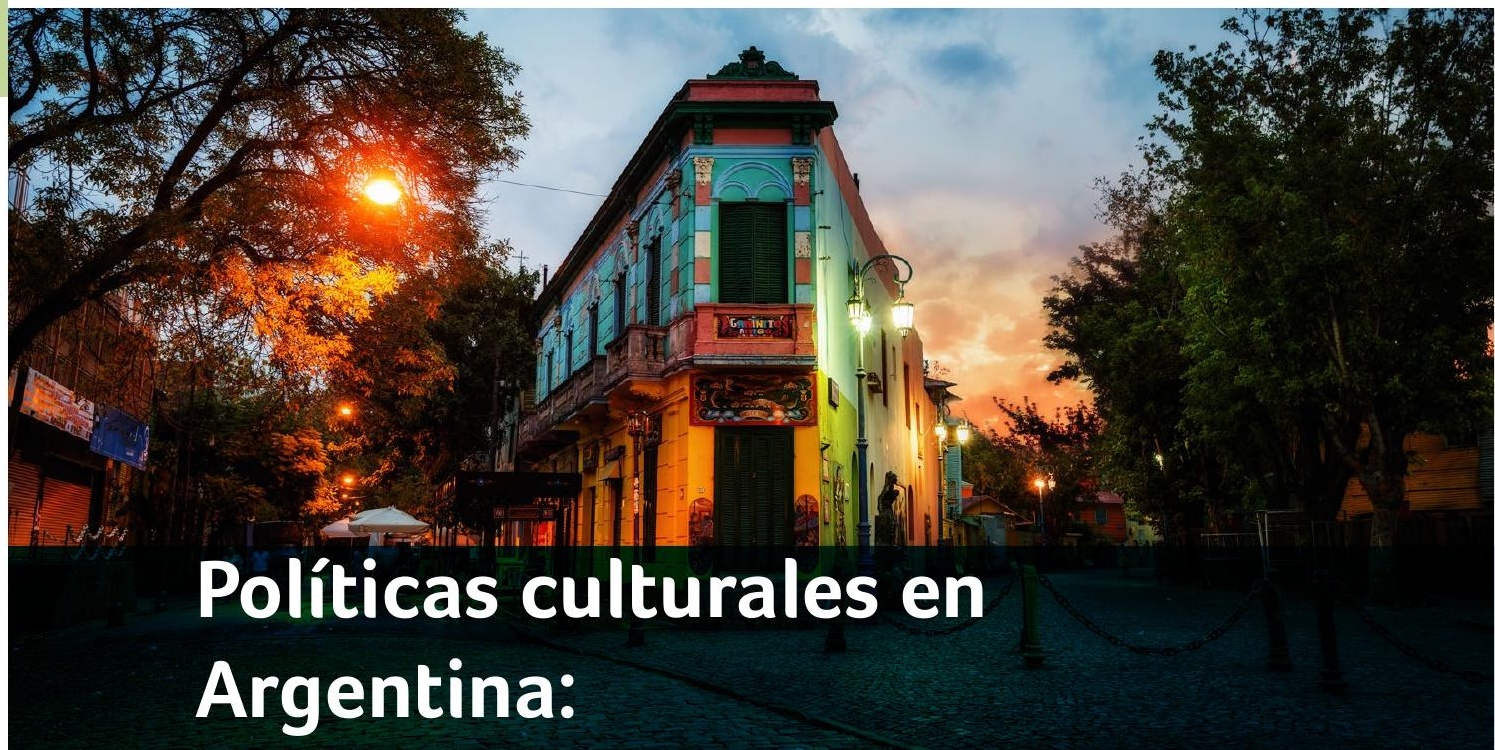

\title{
una revisión en el contexto del Instituto Nacional de Teatro y del Instituto Nacional de Cine y Artes Audiovisuales
}

DOI: https://doi.org/10.21158/21451494.v10.n0.2019.2642

Patricia Relats

Universidad Argentina de la Empresa (UADE) patriciarelatsagmail.com

(c) $\frac{10(3)}{\mathrm{BY}} \mathrm{NC}$

Raúl S. Algán²

Universidad Argentina de la Empresa (UADE)

Consejo Nacional de Investigaciones Científicas y Técnicas de Argentina (CONICET)

raulsantiagoaalgan.com.ar

1 Licenciada en Relaciones Públicas (Universidad de Palermo) y Licenciada en Gestión de Medios y Entretenimiento (UADE), curso de posgrado en docencia universitaria (UADE) y maestranda en Creación y Comunicación Cultural (Instituto Walter Benjamin-CAECE). ORCID: https://orcid.org/0000-0002-0786-857X

2 Licenciado en Gestión de Medios y Entretenimiento (UADE), Magister en Administración de Organizaciones del Sector Cultural y Creativo (UBA), Doctorando en Ciencias Sociales (UBA). ORCID: https://orcid.org/00000001-8659-0889 
Cómo citar este artículo:

Relats, P.; Algán, R. S. (2019). Políticas culturales en Argentina: una revisión en el contexto del Instituto Nacional de Teatro y del Instituto Nacional de Cine y Artes Audiovisuales Revista Comunicación, cultura y política, 10, 36 -69.

DOI: https://doi.org/10.21158/21451494.v10.n0.2019.2642

Fecha de recepción: 09 de marzo de 2020

Fecha de aprobación: 25 de junio de 2020

\section{Resumen}

Política y cultura son fuerzas en tensión constante que influyen en el campo de la gestión y la producción artística. Esta tensión ha sido configurativa en los Estadonación latinoamericanos, en especial en la segunda mitad del siglo XX cuando la cultura ingresa como recurso en las órbitas de la gestión pública. Sin ser ajenos a esta cuestión, los gestores culturales, en específico los productores de cine y artes escénicas, son agentes cuya actividad se ve estrechamente condicionada por el interés público. Con la intención de realizar una aproximación a la relación entre cultura, identidad, política y sociedad, en el presente escrito se abordan las condiciones preliminares que contribuyeron a que en la década de los noventa del siglo XX Argentina tuviera su Instituto Nacional de Teatro y su Instituto Nacional de Cine y Artes Audiovisuales. El teatro y el cine como espejos de una Argentina siempre cambiante son catalizadores de lo cultural que es un tema de permanente revisión. Desde la conformación del Estado en el siglo XIX, pasando por la afluencia inmigratoria europea de comienzos del siglo XX, la cual portó con ella prácticas sociales específicas, hasta los consumidores culturales omnivoros contemporáneos, la cultura es objeto de disputa constante. En este sentido, se profundiza el abordaje incorporando algunas reflexiones sobre los procesos de censura y los proyectos de producción cultural independiente. Corona este recorrido las condiciones actuales de producción independiente y los entrecruzamientos entre lenguajes.

Palabras clave: políticas culturales; gestión cultural; producción artística; agentes culturales; producción cultural independiente; procesos de censura. 


\title{
Cultural policies in Argentina:
}

a review in the context of the National Theatre Institute and the National Institute of Film and Audiovisual Arts

\begin{abstract}
Politics and culture are forces in constant tension that influence the field of management and artistic production. This tension has been a determining factor in the Latin American nation-states, especially during the second half of the 20th century, when culture entered the orbit of public management as a resource. Without being oblivious to this issue, cultural managers, specifically film and performing arts producers, are agents whose activity is closely conditioned by the public interest. With the intention of making an approach to the relationship among culture, identity, politics, and society, this paper addresses the preliminary conditions that contributed to the fact that Argentina had its National Institute of Theater and its National Institute of Film and Audiovisual Arts in the nineties. The theater and the cinema, seen as mirrors of an ever changing Argentina, are catalysts of the cultural factor, which is a subject in permanent revision. From the formation of the State in the 19th century, taking into account the European migratory inflow at the beginning of the 20th century that brought specific social practices, to the contemporary omnivorous cultural consumers, culture has been an object of constant dispute. In this sense, the approach is deepened by incorporating some reflections on the processes of censorship and the projects of independent cultural production. Finally, we wrap this journey with the current conditions of independent production and the crossovers between languages.
\end{abstract}

Keywords: cultural policies; cultural management; artistic production; cultural agents; independent cultural production; censorship processes. 


\title{
Políticas culturais na Argentina: uma revisão no contexto do Instituto Nacional de Teatro e do Instituto Nacional de Cinema e Artes Audiovisuais
}

\begin{abstract}
Resumo
Política e cultura são forças em tensão constante que influenciam o campo da gerência e da produção artística. Esta tensão tem sido presente nos países latino-americanos, em especial na segunda metade do século XX quando a cultura ingressa como recurso nas órbitas do gerenciamento público. Sem ser alheios a esta questão, os gestores culturais, em específico os produtores de cinema e de artes cênicas, são agentes cuja atividade se vê estreitamente condicionada pelo interesse público. Com a intenção de realizar uma aproximação à relação entre cultura, identidade, política e sociedade, no presente escrito abordam-se as condições preliminares que contribuíram a que na década dos noventa do século XX Argentina tivesse seu Instituto Nacional de Teatro e seu Instituto Nacional de Cinema e Artes Audiovisuais. $O$ teatro e o cinema como espelhos de uma Argentina sempre cambiante são catalizadores do cultural que é um tema de permanente revisão. Desde a formação do Estado no século XIX, passando pela afluência imigratória europeia de começos do século XX, a qual trouxe com ela práticas sociais específicas, até os consumidores culturais omnivoros contemporâneos, a cultura é objeto de disputa constante. Neste sentido, aprofunda-se a abordagem incorporando algumas reflexões sobre os processos de censura e os projetos de produção cultural independente. $O$ ponto alto deste percurso são as condições atuais de produção independente e os entrecruzamentos entre linguagens.
\end{abstract}

Palavras-chave: políticas culturais; gerenciamento cultural; produção artística; agentes culturais; produção cultural independente; processos de censura. 


\title{
Politiques culturelles en Argentine: bilan de l'Institut national du théâtre, du cinéma et des arts audiovisuels
}

\begin{abstract}
Résumé
Politique et culture sont des forces en tension constante influençant le secteur de la gestion et de la production artistique. Cette situation exista dans les États-nations latino-américains principalement lors de la seconde moitié du XXe siècle lorsque la culture était considérée comme une ressource appartenant à la sphère de la gestion publique. Partie prenante de cette problématique, les élites culturelles, en particulier les producteurs de films et de spectacles, qui sont des agents dont l'activité est étroitement liée à l'intérêt public, réalisent une approximation de la relation entre culture, identité, politique et société, cet article aborde les conditions préliminaires ayant contribué à la création dans les années 1990, de l'Institut national argentin du théâtre,du cinéma et des arts audiovisuels. Le théâtre et le cinéma, miroirs d'une Argentine en constante évolution, sont des catalyseurs du fait culturel, objet d'un examen permanent. Depuis la formation de l'État argentin au XIXe siècle, en passant par l'afflux d'une immigration européenne au début du XXe siècle entraînant des pratiques sociales spécifiques de la part des consommateurs culturels, la culture a fait l'objet de contestation récurrentes. En ce sens, notre approche intègre certaines réflexions sur les processus de censure et les projets de production culturelle indépendante. Enfin, ce travail analysera les conditions actuelles de la production indépendante et les intersections linguistiques.
\end{abstract}

Mots-clés: politiques culturelles; gestion culturelle; production artistique; agents culturels; production culturelle indépendante; processus de censure. 


\title{
1. Introducción
}

\author{
Todas las teorías son legítimas y ninguna tiene importancia.
}

Lo que importa es lo que se hace con ellas.

Jorge Luis Borges

Se reconocen dos grandes tensiones en Argentina: la de pensar con una lógica europeizante, por la cual se interpreta «qué es lo que nos hace modernos», y otra que implica más estima por las raíces. Estas dos tensiones han manejado implícita o explícitamente los grandes hitos de la historia de nuestro país (Getino, 2005). En esa tensión, el teatro y lo audiovisual no han salido inmunes de la contienda. En este sentido, desde la conformación de las instituciones que se han asentado en todo el territorio nacional desde 1980 en adelante, la conformación de la identidad nacional, el alcance y la ocupación de todo el territorio ha sido a la vez una cuestión política, social y, por supuesto, cultural.

Mientras el teatro en el Río de la Plata nace a finales del siglo XIX, en una carpa de circo, cercano a lo popular y llega a su auge con la oleada de inmigrantes, el cine desembarca con ellos casi seis meses después de que los hermanos Lumiere hicieran la primera proyección (Schoo y Sirven, 2010). En paralelo, ambos sectores producidos han acompañado la historia nacional de manera que confluyen en la vuelta a la democracia que tiene lugar en 1983. Este periodo devuelve al arte la libertad de expresión y la vuelta de los artistas exiliados.

Sin embargo, en la década de los noventa, en el marco del vaciamiento cultural de los medios, la extranjerización narrativa y el achicamiento del Estado vinculado a las políticas neoliberales, aparecen las leyes que promueven el Instituto Nacional de Cine y Artes Audiovisuales (Ley 24.377) y el Instituto Nacional del Teatro (Ley 24.800). Este hito marca, al menos en términos formales, la presencia del Estado y sus consecuentes políticas culturales en todo el territorio nacional, aunque la federalización del acceso a la cultura y la circulación de bienes y servicios culturales sea a hoy un tema pendiente. 
Teatro y cine son lenguajes artísticos diferentes que presuponen una disposición del espectador diversa y promueven, sobre todo, experiencias de apropiación cultural distintas (Tesson, 2012). No obstante, los gestores culturales que llevan adelante proyectos que se insertan en ambos lenguajes muchas veces comparten diseños de producción, aunque tengan especificidades que no se pueden compartir. La comunión en la producción cultural independiente, destinatario final de los institutos que se analizan en este texto, no es actual puesto que, desde la conformación del teatro independiente en 1930 en adelante, los procesos de producción desarrollados en esta línea siempre han alzado la voz en contra de la opresión y la censura (Dubatti, 2012).

En los procesos dictatoriales del siglo XX esto se ve claramente en el ámbito audiovisual con la aparición del Ente de Calificación Cinematográfica, organismo que cortaba los celuloides antes de distribuirlos. En este sentido, las artes escénicas, por depender de la representación y de poner el cuerpo, sufrieron la misma opresión, pero a través de atentados y clausuras.

A partir de lo expuesto, el objetivo es realizar una aproximación a la relación entre cultura, identidad, política y sociedad al abordar, en específico, el Instituto Nacional de Teatro y el Instituto Nacional de Cine y Artes Audiovisuales. Por tanto, en este trabajo se desarrolla un recorrido de literatura sobre la historia cultural argentina en atención a los conceptos mencionados. Se espera indagar con profundidad en la última década del siglo $X X$, en los procesos de censura y en la actividad de los gestores culturales que desarrollan proyectos de producción cultural independiente. Así mismo, se tiene en cuenta el marco de las líneas y los programas que tienen los institutos, desde una mirada estructural y en el intento de poner en tensión la idea de democratización y federalización cultural en el marco de los conflictos contemporáneos. A fin de concluir, se incluyen algunas reflexiones acerca de las implicancias actuales. 


\section{La cultura en las políticas argentinas}

Con la promulgación de la Constitución Nacional de 1853 ese conglomerado de tierras heterogéneas en topografía, clima y pueblos que la habitaban toma la estructura de un Estado-nación en los términos que hoy son conocidos. No obstante, hubo que esperar hasta la anexión de la provincia de Buenos Aires en 1860 al Estado antes mencionado para que este tomara, al menos en las generalidades, su forma actual. Esta tensión entre las antes denominadas Provincias Unidas del Río de la Plata y la Provincia de Buenos Aires tuvieron una corteza y un fondo: la primera fue dirimir a quién pertenecían los ingresos devengados de la actividad portuaria en la aduana; la segunda, decidir un modelo de país federal o unitario ${ }^{3}$, según convenga a la facción, que antes de ser una cuestión política por resolver puede plantearse como una cuestión cultural (Luna, 1997).

Para 1862, cuando se celebraron las elecciones que llevaron a Bartolomé Mitre al gobierno, el primer presidente de todo el territorio nacional, la provincia de Buenos Aires se encontraba incluida en el país y era «su capital provisoria según la ley dictada por la legislatura donde no cedía la ciudad, sino que se invitaba al gobierno nacional a instalarse en calidad de huésped: una sutileza jurídica para evitar la capitalización de la ciudad» (Luna, 1997, p. 102). Se observa, en el discurrir de la historia nacional, así como en la de otros países latinoamericanos, que la relación entre territorio y poder es una puja solo resoluble con guerras. En el caso de nuestro continente esa lucha se trabó en el campo de batalla, primero contra la dominación extranjera y luego contra el caudillismo interno, imponiendo a su paso la configuración de una forma de concebir la cultura (Luna, 1997). 
Esta lucha en torno al territorio es configurativa de las identidades culturales, con su dominancia y su contracultura, que dan forma al ser nacional. Como sostiene Grimson (2011), la alteridad es necesaria para la construcción de identidad porque la configuración cultural,

es la sutura, constantemente reconstruida, de las heterogeneidades inestables pero sedimentadas [...] es el espacio en el cual, a través de hegemonías siempre con riesgos de erosión y de socavamiento, se instituyen los términos de la disputa social y política (p.194).

Así, la presencia de la cultura, término ambiguo, pero constantemente utilizado por actores sociales de todos los tiempos, recorre transversalmente la historia de los Estados contemporáneos.

Cada crisis, con posterioridad a la constitución formal que se ha mencionado, puso en jaque esa identidad cultural. Esta se significa y resignifica de forma constante por medio de leyes, acuerdos tácitos sociales, vinculaciones económicas e instituciones a lo largo de la historiografía nacional. La última gran crisis del 2001 fue para el país un momento de quiebre en el que se revisaron muchos enfoques sobre la cultura y las políticas públicas.

Las políticas, en este sentido, parecen representar las posturas de los gobiernos y ser el reflejo de lo que para ellos es prioritario, así como la construcción ideológica que tienen. En un primer recorte, «las políticas culturales son [definidas como] el conjunto de acciones que impactan en los sectores vinculados a la cultura» (Algán, 2019, p. 107). Pero corresponde abordar la idea desde una mirada propia de nuestro continente.

En este sentido surgen dos definiciones de corte latinoamericanista que se complementan. Por una parte, García-Canclini (2005) define las políticas culturales como el,

conjunto de intervenciones realizadas por el Estado, las instituciones civiles y los grupos comunitarios organizados a fin de orientar el desarrollo simbólico, satisfacer las necesidades culturales de la población y obtener consenso para un tipo de orden de transformación social (p. 72). 
En esta definición se observa que las políticas están orientadas a un fin claro: el desarrollo. Por su parte, Olmos y Santillán-Güemes (2004) las definen como,

\begin{abstract}
un conjunto de intervenciones, acciones y estrategias que distintas instituciones gubernamentales, no gubernamentales, privadas, comunitarias, etc., ponen en marcha con el propósito de satisfacer las necesidades y aspiraciones culturales, simbólicas y expresivas, de la sociedad en sus distintos niveles y modalidades (p. 62).
\end{abstract}

En este caso, la asociatividad y las instituciones son importantes para la definición. Así, no se puede dejar de lado que la idea de una base multicultural se acerca más a cánones democráticos que pensar en una única idea de identidad nacional, en la que una se impone y borra a la otra. Esto, al menos desde una mirada contemporánea, en la cual la búsqueda de las raíces de la identidad nacional es una mezcla cultural entre culturas originarias, primitivas, colonizadoras y migrantes, de manera que el ser nacional podemos definirlo como el resultado de esta convivencia. A partir de esta definición se propone entender por «políticas culturales» aquellas que los gobiernos plasman en leyes, decretos y resoluciones.

En este sentido, la Constitución Nacional Argentina, madre del corpus de leyes nacionales, no es precisa con respecto a qué idea se ha tenido sobre el término. Solo en tres artículos se pronuncia, aunque vagamente, al respecto. El primero es el artículo 41 y lo hace refiriéndose a «la preservación del patrimonio natural y cultural» (Constitución de la Nación Argentina, 1999, p. 30) como una obligación del Estado. En el segundo, el artículo 75, dota al congreso para «dictar leyes que protejan la identidad y pluralidad cultural, la libre creación y circulación de las obras del autor; el patrimonio artístico y los espacios culturales y audiovisuales» (Constitución de la Nación Argentina, 1999, p. 45). Finalmente, en el artículo 125 lo hace al permitir que las provincias puedan conservar organismos para promover «la educación, la ciencia, el conocimiento y la cultura» (Constitución de la Nación Argentina, 1999, p. 76).

Es válido remarcar que una postura exclusiva o una sola definición de cultura o de políticas culturales no es perdurable en el tiempo. En este sentido, la Constitución puede operar como un marco de relevancia y referencia sobre el cual las leyes y los decretos lo anclen a un espacio-tiempo y a una perspectiva sobre dichos elementos. 
En la década del noventa,

la conformación de conglomerados multimedia en la Argentina formó parte del proceso globalizador de la cultura en Occidente [...] consecuentemente se restringe el gasto en servicios sociales: entre ellos, el financiamiento de programas educativos y culturales, y la inversión para investigaciones científicas (Wortman, 2005, p. 253).

Es en ese contexto que se da la reforma constitucional de 1994. En ella se incorporó el denominado bloque de constitucionalidad, el cual emana del artículo 75 mencionado. Este artículo, enmarcado en la segunda parte que hace referencia a las autoridades de la nación, establece las atribuciones del Congreso. Este orden constitucional está integrado, en primer lugar, por los tratados internacionales, como, por ejemplo, la Declaración Universal de Derechos Humanos y el Pacto Internacional de Derechos Económicos, Sociales y Culturales entre otros. Integrar los tratados internacionales al bloque constitucional es una forma de alinearse con el mundo, frase muy repetida en la década de los noventa en la Argentina.

\section{La conformación de las identidades culturales a través de las políticas públicas y el valor simbólico}

Appadurai (2001) plantea que la Modernidad armó un mito a partir de sí misma: la de romper con el pasado, presentándose como novedosa. El cine nace con la Modernidad y, en esta cosmovisión que no cree en las fronteras porque acorta distancias, es que se presenta el mismo relato para espectadores desterritorializados. Apoyada en los medios de comunicación de masas, la imaginación, la vida cotidiana y las formas de construirse a uno mismo son moldeadas por estos ejemplos. Por otra parte, se enfoca en el rol de la imaginación en cuanto a que, con la penetración de estas narrativas, pasa de ser individual a ser global. Imaginar, para el autor, no es liberador, sino que están en juego una serie de mensajes, símbolos y estructuras en tensión que permiten vincular la rutina cotidiana con lo global. Se convierte en otro 
proceso de sociabilización que pone en jerarquía cómo integrarse al mundo. Por su parte, las artes escénicas, si bien nacen con anterioridad al periodo que se describe, se ven influenciadas por esa cosmovisión del ser humano y del mundo. Existe un tipo de drama que «guarda relación con los principios basales de la Modernidad: es la poética de mayor representación histórica de la Modernidad, tal vez la contribución más específica y original que la Modernidad ha realizado a la historia del teatro mundial» (Dubatti, 2009, p. 22). Si bien esta forma teatral se ubica entre el siglo XVIII y el XIX, se desarrolla aún en la actualidad. Pero, independientemente de su ubicación histórica, son sus características de identidad las que nos interesan. Por un lado, es marco de un nuevo registro de actuación donde «empieza a concebirse el personaje como un "reflejo" de los hombres tal como actúan en la vida pública» (Dubatti, 2009, p. 32). Este nuevo canon de actuación, que desplaza el método recitativo propio del teatro medieval e isabelino, es deudor, a su vez, de una forma de escritura literaria también realista.

En esta línea, cabe mencionar que las representaciones sociales de la imaginación reconocibles como narrativas cuentan con una regulación de la base moral del entorno. Retoma, como base de esta genealogía, los mitos y leyendas, reconociendo su esfuerzo por separarse de la vida cotidiana y trascender. Los individuos, aun en las sociedades más simples, encontraron en sus sueños una forma de reorganizar su vida social, cosa que para Appadurai (2001) ya no es liberadora, sino reflejo de una falsa universalidad. En la misma línea es dable considerar que «la lógica del capitalismo contemporáneo remite a transformaciones en lo macroeconómico, como también en la dimensión cultural de la vida social y económica, en la reorganización del campo cultural y en la emergencia de nuevas subjetividades» (Wortman, 2005, p. 253-254). Así, tanto a la esfera económica como a la política las atraviesa lo cultural como fenómeno social.

Como se ha mencionado, García-Canclini (2005) toma como materia prima para las políticas culturales la idea de «desarrollo simbólico». Bajo esta premisa, la modernidad en cuanto precepto se plasma en el pensamiento y en las instituciones como único resultado de modelos de globalización. Appadurai (2001), de hecho, profundiza sobre esto al alinear su perspectiva sobre la imaginación con la Escuela de Frankfurt y expone así que la producción seriada atrofia la imaginación e impone un modelo de sueño para quienes están expuestos a esos mensajes. En este sentido, Williams (1988) 
presenta una aproximación al concepto de cultura en la que la presenta en esa tensión constante, ya que está en permanente diálogo con otros conceptos: relacionar cultura con economía, cultura con sociedad, a fin de aproximarse a influencias y alcances. A fin de analizar esto, presenta a la cultura en tres esferas: emergente - experimental y periférica —, residual —obras anteriores revisitadas por el público contemporáneo-y dominantes -instituciones naturales y necesarias-. Estos ámbitos de circulación de bienes artísticos, plantea el autor, son dinámicos y están en constante movimiento.

Ahora bien, esos ámbitos de circulación constituyen una suerte de mercado simbólico en el que confluyen la producción cultural y la recepción. En este sentido, «tal vez no haya ningún otro campo en el que la relación entre la economía y la cultura sea más directa que en el ámbito de las políticas públicas» (Throsby, 2001, p. 153). Esto es así porque la cultura, al igual que otras áreas productivas de una nación, genera un valor económico y simbólico que debe ser de interés para los gobiernos. No obstante, en el caso de Latinoamérica, en general, y de Argentina en particular, se observa que las relaciones sociales vinculadas a los aspectos económicos y políticos ni han conformado un mercado cultural de impronta burguesa ni han redundado en la generación de una producción intelectual autónoma (Wortman, 2005). Aun así, la relación entre las variables que se mencionan aparece en las leyes que materializan las políticas culturales, tal como se analiza más adelante.

La cultura agrega valor a la sociedad de diferentes formas (Bonet, 2007; Getino, 2007; Throsby, 2001). Ese valor puede ser económico, en el caso de la generación de divisas o de empleo, pero también simbólico, como lo puede ser, por ejemplo, el incremento del capital cultural de los ciudadanos. Independientemente de la categorización a la que llegue, debe entenderse esta actividad como una actividad esencial por dotar de conocimiento a la ciudadanía de una nación y ser una fuente de empleo. Además, es una de las pocas actividades que genera un impacto positivo en otras que le son conexas, como, por ejemplo, la gastronomía, el turismo o la indumentaria. En este sentido, «los estudios que se han realizado sobre la demanda cultural demuestran cómo el consumo cultural es adictivo. Quien más libros tiene es quien más libros compra» (Bonet, 2007, p. 23). Esta afirmación demuestra que la utilidad marginal de un consumo cultural no sigue un patrón lógico que responda a las premisas del mercado, sino que es un tipo de práctica más bien emocional. 
Así, entonces, aparece la dificultad de valorar las actividades artísticas y culturales. Es decir, entender que «cuando uno compra un libro [...] posiblemente está buscando los contenidos que lo van a entretener o van a servir a su desarrollo técnico, educativo o el que fuere» (Getino, 2007, p. 80). De esta manera, un libro, para seguir el ejemplo que brinda el autor, tiene un valor que deviene del proceso de manufactura de la creación del soporte papel y un valor simbólico que es la obra contenida en sus páginas. Esa dualidad emerge en el resto de las interpretaciones sobre el valor.

En razón a lo expuesto, Argentina, así como otros países de América Latina, se encuentra en una instancia en la que su sector cultural puede entenderse como motor de inclusión, integración y difusión de la cultura de la región. Esto es así porque «para servir a los intereses de la economía y de la cultura, la política cultural debería tener en cuenta tanto el valor económico como el cultural de los resultados que pretende alcanzar» (Throsby, 2001, p. 165). Las políticas culturales y las acciones de Estado orientadas al fortalecimiento de este sector muchas veces llegan mal y tarde a quienes las necesitan. Así, el potencial crecimiento al cual no llega el área se vuelve una dificultad para sí misma porque se estanca y refuerza, de forma indirecta, la idea antigua según la cual el arte y la cultura son deficitarios.

\subsection{Las culturas nacionales}

Construir una identidad nacional ha sido el gran proyecto político de la Generación del $80^{4}$. Este grupo político, fiel a muchos estándares de la Modernidad europea, materializó las grandes instituciones públicas que le dan forma al Estado-nación como hoy lo conocemos. Las presidencias de este periodo han construido escuelas, ministerios, correos y ferrocarriles que han sido claves para poblar el territorio nacional y estipular, a través de guerras, las fronteras. En palabras de Bauman (2013), «la cultura [en la Modernidad] se asemejaba ahora a un mecanismo homeostático: una suerte

\footnotetext{
4 Este es el nombre que recibe la élite conservadora que gobernó la República Argentina entre 1880 y 1916 . Se trataba de un conjunto de dirigentes intelectualizados y de descendientes, en su mayoría, de familias patricias. Se los vincula a la Generación del 37 que fue la encargada de pensar y concebir en términos intelectuales el ser nacional, mientras que la del 80 llevó a la práctica ese pensamiento armando las instituciones que hoy son organismos públicos.
} 
de giroscopio que protegía al Estado-nación de los vientos de cambio y de las contracorrientes, [...] a mantener el barco en su rumbo correcto» (p.16). Es decir, en este periodo, la cultura se consideraba un factor aglutinador y homogenizador de identidades donde una visión dominante, burguesa y europeizada primaba sobre la diversidad cultural de los pueblos originarios. Esto es así porque la Modernidad sostiene una única posible identidad y no identidades que conviven, como se mencionó.

Problematizar la identidad, en palabras de Hall (1996), es cuando uno no sabe quién es. Es en este punto que la Modernidad entra en crisis y da paso a los tiempos contemporáneos: si hay una única visión o perspectiva sobre lo que implica una identidad nacional, esta no estaría reflejando a los habitantes de un mismo suelo. En este contexto se inserta la visión de organismos internacionales que reconocen cómo «toda cultura tiene una dignidad y un valor que deben ser respetados y protegidos» (Unesco, 1966). O más bien, como sostiene Bauman (2013), «las relaciones culturales ya no son verticales sino horizontales: ninguna cultura tiene derecho a exigir la subordinación, humildad o sumisión de otra por la simple consideración de su propia superioridad o su carácter progresivo» (p. 37). Este es el principio de la diversidad cultural que hoy en día está instalada como discurso, pero que hace un par de décadas atrás no era recurrente en el pensamiento local. Es válido aquí retomar el concepto de las esferas culturales de Williams (1998) mencionadas: la cultura es un compuesto de tensiones que dialogan y, de la misma manera, los agentes propician dicho movimiento. La identidad cultural, al no ser cuestionada hasta que no se encuentra en crisis, dependerá de la mirada sobre los espacios culturales.

Olmos y Santillán-Güemes (2004), por su parte, plantean que aún estamos obsesionados con definir qué es nuestra identidad cultural porque nos aterroriza la idea de diversidad. En esta misma línea, no habría una única manera de ser nacional. No debe dejarse de lado que la noción de lo que es ser criollo tiene su génesis en la perspectiva de intelectuales, mientras la modernización dejó atrás al gaucho, que ha pasado de un independiente, a un forajido o un alienado ${ }^{5}$. Bhabha (1994), en este mismo sentido, estipula que lo

\footnotetext{
5 El Martín Fierro, una de las obras más reconocidas del género de las gauchesca, lo escribió José Hernández mientras huía de una peste encerrado en un hotel. No convivió con los gauchos y sus costumbres, por lo cual se lo considera nativismo.
} 
colonial abarca un sinsentido en el que su postura arcaica rechaza la otredad cultural, dividiéndolos en puntos opuestos: caos/urbanidad, civilización/ barbarie. El valor de las palabras es la capacidad de plantear dichas diferencias: la cultura es el resultado de la mezcla de ambas. Por esto, culturalmente, no es relevante seguir hablando de federales versus unitarios, pero sí desde el punto de vista de la gestión y la cantidad de proyectos que se concentran en ciertos centros urbanos, entorpece la circulación de bienes y productos culturales y favorece a unos sobre otros ${ }^{6}$.

De esta manera, en Argentina se observa que, construido el ser nacional, la gran y hegemónica identidad cultural del Río de la Plata a finales del siglo XIX, comienza a sucederse, entrado el siglo XX, un proceso de reconfiguración. Este proceso puede verse reflejado en las áreas culturales que se analizan y el motor de este es la afluencia inmigratoria que tiene a Buenos Aires como epicentro. Aquí no solo se hace referencia a la inmigración externa sino también a la confluencia de criollos que, junto con esta, conforman el suburbio como un espacio propio. Al respecto cabe recordar que con la llegada de vastas olas inmigratorias se creó en la ciudad puerto de Buenos Aires un nuevo territorio suburbano en el que se comenzaron a negociar los paradigmas culturales que traían los recién llegados con el fin de generar una nueva identidad (Pellarolo, 1997). Otras tensiones y cambios sociales, como, por ejemplo, el arte de resistencia durante la última dictadura cívico-militar o el vaciamiento cultural de los noventa y la posterior crisis del 2001, hacen que estas esferas que componen la cultura sean un sinfín de tirantez.

Roberto Arlt ${ }^{7}$, desde la prensa de la época, en 1932, publica en un artículo que, de alguna forma, refleja la visión que se tenía sobre la clase media argentina. En ese artículo, el periodista discutía con el líder del Partido Comunista e indicaba que «de cien proletarios [...] 90 ignoran quién es Carlos Marx [...] pero 90 pueden contestarte en qué estilo daba besos Rodolfo Valentino» (Arlt, 1999). Los artículos culturales de Buenos Aires de las décadas del veinte y del treinta «[presentan] una cultura de mezcla, donde coexisten elementos defensivos y residuales junto con los programas renovadores; rasgos culturales de la formación criolla al mismo tiempo que un proceso descomunal de importación de bienes, discursos y prácticas simbólicas» (Sarlo, 1988).

\footnotetext{
6 Se amplía sobre los aspectos económicos en el análisis de las leyes y las reflexiones finales.

7 Periodista argentino de gran relevancia nacido en Buenos Aires en 1900. Fue, además, autor y dramaturgo de muchas piezas teatrales estrenadas por las compañías independientes de la década del treinta.
} 
Al igual que Appadurai (2001), con las consecuencias que plantea para la imaginación la producción simbólica seriada, Arlt (1999) consideraba que era esa cultura masiva la que dormía la posibilidad de desarrollar una consciencia de clase, pero lo que no pudo ver es que en realidad era el escapismo lo que ayudaba a mitigar el dolor: el jazz y las luces de Hollywood. Es por esto por lo que los productores locales se fueron volcando a hacer historias con un estilo similar y sabor local. Como resultado, se pueden encontrar filmes a la manera de Hollywood, con grandes estudios y un star system consolidado, el melodrama como género más popular en cuyo centro de la trama presentaba una idea de justicia social: el pobre era siempre bueno y el protagonista y el rico es tratado como deshonesto, traicionero y mala persona (Karush, 2013).

Ahora bien, en este punto, las maquinarias culturales argentinas no eran exclusivamente el cine y el teatro, como se viene mencionando hasta ahora. Otras actividades sociales eran tanto o más susceptibles de ser abordadas desde las políticas culturales y en relación con la afluencia inmigratoria. Se puede detectar también la radio, los cabarets donde se bailaba tango o en el deporte un fenómeno similar. Como sostiene Alabarces (1998),

\begin{abstract}
si lo nacional se construye en el fútbol, hay que explicar el tránsito de la invención inglesa a la criollización [...] el par nosotros/ellos encuentran su expresión imaginaria en un estilo de juego [...] [de] gran capacidad productora de sentido (pp. 7-8).
\end{abstract}

Es decir, en el proceso de conformación de nuestra identidad, diferentes maquinarias como, por ejemplo, las mencionadas, han sido definitorias para arribar a ser lo que somos. En este sentido, el proceso que el autor denomina criollización está estrechamente vinculado a la apropiación cultural de la práctica y su resignificación en el propio sistema de valores de los ciudadanos locales.

Más cerca de la actualidad y tras la última dictadura cívico-militar que tuvo su ocaso con la guerra de Malvinas en 1982, las políticas culturales estuvieron orientadas a la revalorización de los derechos humanos (Dubatti, 2012). Con la transición democrática del gobierno de Raúl Alfonsín -1983-1989la acción cultural comienza a resignificar hacía los derechos sociales y la ampliación ciudadana, proceso que encuentra su punto culmen en la nueva Constitución de 1994. Así «el vínculo entre cultura y política en la Argentina 
adoptó nuevas significaciones en los años optimistas de la transición democrática, donde se hicieron innumerables balances de la destrucción de la cultura y la educación» (Wortman, 2005, p. 253). No obstante, da la sensación de que mientras en América Latina el componente mestizo está presente en la reconstrucción del imaginario social, en Argentina esa meta es difusa puesto que estas identidades locales son minoritarias y están desplazadas del discurso estatal.

\section{Impacto de las políticas culturales en la producción cultural: el caso de la ley nacional de Cine (Ley 24.377) y la ley nacional de teatro (Ley 24.800)}

Con el fin de arribar a un nivel de análisis más operativo sobre las políticas culturales en Argentina, se propone, a continuación, una descripción de las leyes que regulan las actividades vinculadas a la producción audiovisual y a la producción escénica. Ambas ven la luz en la década del noventa con posterioridad a la reforma constitucional de 1994 y son, de alguna forma, deudoras de la cosmovisión sobre la cultura que se ha explicado.

Una de las tesituras recurrentes en las políticas culturales de esta época es la necesidad de federalización cultural que un territorio tan extenso y heterogéneo como el argentino demanda. Si bien esa discusión pudo haber mutado en la actualidad, como se intentará abordar más adelante, es destacable puntualizar en el espíritu de la época porque subyace en los programas que se describen y arraiga en los artistas que colaboraron con la sanción de la ley y la puesta en marcha del instituto tanto de cine como de teatro. Los promotores de su creación sostenían que «el teatro no puede pasar solo por la calle Corrientes. El teatro argentino comienza en Tierra del Fuego y termina en Jujuy. Con este instituto tenemos que generar un hecho verdaderamente federativo» (Concepción Roca, citada en El Instituto Nacional del Teatro, en marcha, 7 de abril de 1998). 
La Ley Nacional del Teatro ${ }^{8}$ de 1997 representó para la comunidad artística una conquista sin antecedentes. En palabras del actor Pepe Soriano, el logro fue titánico porque «durante estos 50 años de espera, hemos protestado y luchado tanto. Salió, y en esta salida hay mucha gente anónima [...] ahora, poner la rueda en marcha va a ser muy duro» (El teatro cumple el sueño del instituto propio, 30 de septiembre de 1997).

Cabe mencionar que para esta época tanto el sector teatral como el audiovisual ya contaban con el Fondo Nacional de las Artes, el cual es un instituto nacional creado en 1958 y cuya labor se orienta al sostenimiento de la producción artística. No obstante, tener un Instituto Nacional de Teatro implica poder defender la política cultural y la estrategia de gestión pública con sus rasgos particulares. Es decir, la importancia de tener una ley nacional de este tenor estriba en que se delimite la definición de actividad teatral, la creación del instituto y el régimen económico y financiero de la actividad teatral. Por otra parte, el cine tuvo una ley anterior, sancionada durante la última dictadura cívico-militar, en la cual el espíritu era utilizar la cinematografía con fines de promoción política - el instituto que queda inaugurado después de la sanción de la ley dependía de la Secretaría de Turismo- y el cine permitía no solo una llegada a todo el territorio nacional, sino también control total sobre el mensaje (Delupi y Vibes, 2006).

En lo que refiere al control del Estado sobre la cultura, es evidente que teatro y cine presentan modos de producción cultural diferentes y, por tanto, diferentes mecanismos de intervención. Esta censura directa, si bien no se ve reflejada en las leyes que se analizan por ser concebidas en democracia, fueron antecedentes directos de estas por haber impregnado la dinámica cultural de la dictadura cívico-militar que se mencionó. Las artes escénicas, al ser un evento vivo, permitían que las manifestaciones contra la dictadura fueran más notorias, más públicas y con un limitado acceso a detener la obra hasta que no sucediera un hecho que se considerara que estaba dirigido en contra de ellos 9 .

8 Véase la ley completa en http://www.infoleg.gob.ar/infolegInternet/anexos/40000-44999/42762/texact.htm. 9 Cabal ejemplo de esto fue el ciclo Teatro Abierto que comenzó en 1981 y tuvo dos ediciones más en 1982 y en 1983 durante el gobierno de facto. En su primera edición, un incendio destruyó el teatro Picadero donde se estaba llevando a cabo el evento. No obstante, los realizadores continuaron con la actividad en otra sala. 
El cine podía, de alguna manera, contralarse previo al estreno y durante los rodajes. Si a esto se suma la identificación de algunos personajes en la cultura popular como, por ejemplo, Palito Ortega, o el cine de Alberto Olmedo y Jorge Porcel, que fueron utilizados por el gobierno de facto para instalar películas livianas en las pantallas a espaldas de lo que sucedía socialmente, termina por provocar una división del público nacional. No existe un cine que refleje y lo que refleja es para esconder lo de adentro. Aquellas películas que fueron censuradas como La hora de los hornos o La Patagonia rebelde, fueron las primeras en proyectarse una vez que volvió la democracia y, a modo de homenaje, hasta el día de hoy al menos una de las dos se pasa en los colegios secundarios (Peña, 2012).

El primer presidente del instituto, en 1978, afirmó que su idea era,
ayudar económicamente a todas las películas que exalten valores espirituales, cristianos, morales e históricos o actuales de la nacionalidad o que afirmen los conceptos de familia, del orden, de respeto, de trabajo, de esfuerzo fecundo y responsabilidad social, buscando crear una actitud popular, optimista en el futuro (García, 3 de agosto de 2018).

Un cine silenciado que también marca otra división: si la imaginación queda moldeada, como señala Appadurai, en una falsa idea universal, no queda posibilidad de una identificación con las narrativas que busquen lo local.

El repudio a lo sucedido en la última dictadura cívico-militar argentina hace que la modificación de esa ley también cambie en muchos enfoques, excepto en uno: el interés por vincular cine y turismo. En este sentido, por más que el instituto migrara de su original filiación a la Secretaría de Turismo al ahora Ministerio de Cultura, la búsqueda permanente de presencia en festivales internacionales, de que las producciones extranjeras vengan a rodar al territorio nacional y la constante mención de las regiones subnacionales como locaciones evidencian que su afán por seguir promoviendo el turismo receptivo sigue intacto. Incluso esta premisa no se ha visto modificada, dada la condición de ser un ente autárquico y no gubernamental, situación que le da mayor libertad. 
De esta forma, se observa una intencionalidad política en el uso del cine como industria cultural: buscar encantos urbanos con miras a hechizar mercados internacionales. En lo que se refiere a las artes escénicas, al no haber una ley previa la sanción de esta se presentó como una novedad. Actualmente, el Cine Gaumont, sala de exhibición cinematográfica reconocida como el KM 0 del INCAA, cerrará por dos meses debido a obras que mejorarán su estructura. El cine local está alarmado porque sin la pantalla del instituto muchos filmes podrían no tener estreno. Luis Puenzo, el actual presidente del instituto brinda entrevistas hablando de su amor al cine y dice que es peor para una película estrenarse en un contexto desfavorable. Así, ser testigos de las repercusiones devengadas de la actividad de ese espacio brinda una pauta clara de lo vital que es el INCAA para la producción nacional cinematográfica (Cinelli, 2020).

\subsection{Precisiones de las leyes que impactan en la gestión cultural}

En lo que se refiere a definiciones se observa que la actividad teatral se entiende como «toda representación de un hecho dramático manifestada artísticamente a través de distintos géneros» (Ley Nacional 24.800 de 1997). Estipula, además, que esa representación debe ser de carácter público, incluida en alguna de las teatralidades existentes y debe implicar la «participación real y directa de uno o más sujetos compartiendo un espacio común con su auditorio» (Ley Nacional 24.800 de 1997). Describe también a quienes participan en la representación escénica, agrupándolos según tengan una relación directa con el público, con la realización artística o indirecta con el hecho teatral. Con respecto a los espacios teatrales, reconoce los que tienen hasta 300 localidades como los prioritarios a la hora de definir las acciones del instituto. Lo que no define la ley, que es, de hecho, un área de vacancia actualmente muy criticada, es la formación de espectadores y el desarrollo de públicos, así como el teatro comunitario ${ }^{10}$.

\footnotetext{
10 Esta forma teatral la desarrollan los vecinos teniendo como público objetivo a su comunidad. Trabaja desde la concepción del derecho al arte que todo ser humano tiene, ancla en un territorio barrial y crea de manera colectiva a partir del trabajo de quienes coordinan los grupos, quienes sí son profesionales del arte. Los grupos son abiertos, numerosos y heterogéneos en sentido amplio. La identidad y la memoria son pilares de esta práctica, así como la fiesta y la celebración. Construye desde el nosotros como lenguaje discursivo y su práctica genera transformación social (A. Hanna, comunicación personal, 2020).
} 
En el caso del cine, por otra parte, la ley no lo define solo como una actividad, sino también como un producto cultural acabado. Define a la película como:

Todo registro de imágenes en movimiento, con o sin sonido, cualquiera sea su soporte, destinado a su proyección, televisación o exhibición por cualquier otro medio. Quedan expresamente excluidas del alcance del presente artículo: las telenovelas y los programas de televisión (Ley Nacional 24.377 de 1994).

Es llamativo este último punto diferencial, en consideración a que los melodramas televisivos han sido un sello de venta internacional de contenidos argentinos y esta ley los rechaza de forma categórica. Por otra parte, otros términos considerados como parte de la actividad definen al editor de videogramas grabados como el poseedor de los derechos de comercialización, al distribuidor de videogramas, a quien, por cesión de derechos por parte de los editores puede comercializarla, y al videoclub como el establecimiento dedicado a la comercialización minorista. Se puede identificar, así, que la ley tiene intención de profesionalizar y fortalecer la cadena de valor de la industria. De igual forma, en el artículo 16 se indica expresamente el tiempo de exhibición en diferentes ventanas:

Las películas de largometraje no podrán exhibirse por televisión o editarse en videocassette, en el territorio argentino, salvo autorización previa del instituto, antes de haber transcurrido seis (6) meses de su primera exhibición comercial en el país. No quedan comprendidas aquellas películas cuyo destino de exhibición es exclusivamente televisivo o videográfico (Ley Nacional 24.377 de 1994).

El gran conflicto que presenta esto es que el streaming ha acortado y afectado la distribución. En este contexto, esta cadena de valor se ha visto ajustada y modificada a una celeridad que no permitirían los seis meses que pide la ley, con lo cual el INCAA se vería en la obligación de hacer excepciones o incumplir definitivamente este artículo. No es menor remarcar que ya en el caso de la exitosa película El secreto de sus ojos de Juan José Campanella, en el momento en el que el filme estaba en cartelera, se editaron los DVD de la película a precio 
muy bajo con el fin de promover la compra y no la piratería ${ }^{11}$. Las llamadas OTT $^{12}$ no generan el ingreso de valores que equipare a la sala y no se conocen casos de multas a salas por no respetar la cuota de pantalla estipulada por la ley de cine.

En lo que a su organización se refiere, el INT, dependiente del Ministerio de Cultura $^{13}$ de la Nación, cuenta con un director ejecutivo que es designado por el Poder Judicial. Tiene, además, un Consejo de Dirección integrado por el director ejecutivo, un secretario general, un representante del Ministerio de Cultura de la Nación, cuatro del quehacer teatral y uno por cada región del país - Centro, Litoral, Noreste Argentino, Noroeste Argentino, Cuyo y Patagonia-. A su vez, cada provincia tiene un representante y el instituto cuenta con programas de acción específicos desde los que se trabajan políticas culturales de interés. Por su parte, el INCAA se crea en la reforma de 1994, en la cual se define que su lugar depende del Ministerio de Cultura como un ente autárquico. De todas maneras, uno de los elementos más atractivos de la reforma de la ley está vinculada a la composición del Gobierno y su administración: director y subdirector, asamblea federal y el consejo asesor. De igual forma, se invita a una mirada más amplia en el momento de juzgar una obra como de interés. La composición de la administración implica un equilibrio entre Estado e industria que ayudaría a cuidar los intereses del sector.

Uno de los deberes a resaltar del director del INCAA, como responsable de la gestión, es la de acrecentar la difusión del cine nacional en el ámbito internacional. Esto se relaciona con algunos temas importantes, como, por ejemplo, que Argentina no cuenta con una cantidad importante de espectadores en cuanto a disponibilidad de salas fuera de los centros urbanos

\footnotetext{
11 Cabe aclarar que la televisión no tuvo una ley propia hasta que se sanciona en el 2009 la ley de medios audiovisuales. Hasta esa fecha, la TV se basó en la ley de radiofonía y cinematográfica para encontrar una regulación. A partir del 2009, el INCAA cuenta también con programas de fomento para series y TV. Actualmente, dicha ley se encuentra derogada.

12 La sigla OTT significa over-the-top e implica la libre circulación de contenido en plataformas digitales. Estas pueden ser gratuitas, como es el caso de Cuevana; mixtas, como el caso de Cine.ar o pueden ser por suscripción fija y acceso al catálogo completo, como HBO o Disney Plus.

13 Los vaivenes de los sucesivos gobiernos de la República Argentina han hecho pendular la figura del ministerio de cultura entre esta categoría y la de secretaría desde 1983 a la fecha. Sin ir más lejos, el gobierno de Mauricio Macri —2015-2019—, sobre la segunda mitad de su gestión, degradó el Ministerio de Cultura de la Nación a secretaría, mientras que el actual presidente, Alberto Fernández, lo volvió ministerio de nuevo en una de sus primeras acciones de gobierno.
} 
y la asistencia del público a estas. En el 2018, cayó tres millones de tickets más que en el 2017 y presenta una tendencia a la baja desde el $2014^{14}$. Las salas están concentradas, en su mayoría, en las ciudades, y aun así la llegada de oferta es menos variada cuanto más se aleja de Buenos Aires. El cine argentino no tiene una particular recepción en el mercado interno ${ }^{15}$, a menos que cuente con estrellas de un star system televisivo o que haya tenido una aceptación relevante en festivales internacionales. Tras la crisis del 2001, Argentina se encontró con otra particularidad en el sector audiovisual: profesionales capacitados y con mucha experiencia, locaciones atractivas por la arquitectura ecléctica de la ciudad y los costos de producción eran significativamente más bajos. El auge de los servicios de producción hizo que la mayoría de las óperas primas de toda una camada de directores fuera en otro idioma. En este contexto, el cine de género fue uno de los más prolíficos.

En el 2003, el entonces director del INCAA, Jorge Álvarez, señala: «Quieren establecer un convenio con la Secretaría de Turismo y el Instituto de Cine para poder vender material afuera y que vengan a filmar acá» ${ }^{16}$ (como se cita en Olmos y Santillán-Güemes, 2004). Lo anterior habla del reflejo de la situación y cómo esto presentó una salida económica. Sin embargo, muchos de esos proyectos no califican para los fomentos del instituto. ¿Por qué volvemos al turismo y no hablamos de la cultura? El hecho de que el INCAA cambie su dependencia habla también del lugar que ocupa la cultura en determinado contexto y se puede retomar a Williams (1988) con sus tensiones y esferas por una simple razón: no pueden establecerse parámetros claros de hacia dónde va la política sin tener un consenso sobre la definición de cultura.

\footnotetext{
14 En el momento de escribir este artículo, la película con más espectadores esta semana corta 608582 tiquetes acumulados en casi una semana de estreno, en 281 pantallas (Batlle, 2018).

15 El informe sobre consumos culturales del Sinca (2017) establece que el 35 \% de la población fue al menos una vez al año al cine. De ese $35 \%$, la mitad asistió trimestralmente, pero afirma que vio al menos una película argentina en el año. Estos datos demuestran que no hay una cantidad de tickets alta en salas y que las elecciones de películas en un bajo porcentaje son de origen nacional. En el año de la muestra del Sinca se estrenaron 217 películas (Ministerio de Cultura, s. f.).

16 Si bien renunció entre un mar de denuncias por aplicar cuestionablemente créditos y subsidios del instituto, en el 2019 repetía esta misma intención como presidente del Polo Audiovisual de Córdoba, demostrando que no es netamente un tema de coyuntura, sino una constante obsesión del sector (Secretaría de Cultura, 2019).
} 
4.2 Las acciones de los gestores culturales en el marco de las políticas existentes

El debate sobre la cuestión social en Occidente, históricamente, ha estribado entre agencia y estructura. Mientras que la idea de agencia remite al sujeto como actor social, la estructura remite a fuerzas sociales que condicionan el accionar del individuo (Mendes-Calado, 2019). Para la cuestión que en este artículo se trata, el agente es el gestor cultural que con su trabajo desarrolla proyectos desde el ámbito público o privado. Por su parte, las políticas culturales funcionan como estructura contenedora de su accionar al funcionar como mutuos condicionantes de la actividad cultural.

En este sentido, como afirma Fuentes-Firmani (2019), el gestor cultural es un agente que puede impulsar el debate que propicie nuevas políticas de desarrollo de la actividad. Es decir, como agente de un campo intelectual su accionar condiciona y es condicionado. En esa dicotomía se insertan las políticas culturales como instancias de mediación. A su vez, el autor define que el objeto de estudio de la gestión cultural lo comparte con las políticas y los modelos de gestión. Esto debido a que la disciplina cuenta con una delimitación intelectual y empírica como para tener la solidez necesaria de cara a ser un espacio de reflexión autónomo. Por esta razón, profesionalizar la actividad redunda, como también sostiene Bayardo (2019), en el fortalecimiento del sector y sus economías conexas.

Cabe definir la gestión cultural como una actividad que da origen a algo, que produce hechos sociales. Así, entonces, ¿si se considera que gestamos la cultura, es porque somos parte de aquella cultura a la que estamos dando origen? Caracterizada la gestión, no sería posible hacer lo mismo con el término «cultura» por ser un concepto tan brumoso. No obstante, cabe mencionar que cuando se habla se referencia al «conjunto de fenómenos que contribuyen, mediante la representación o reelaboración simbólica de las estructuras materiales, a comprender, reproducir o transformar el sistema social» (García-Canclini, 1983, p. 19). Aunque cultura no es en sí mismo un concepto excluyente, sí es abordable desde las diversas ciencias sociales, como, por ejemplo, la antropología o la sociología. Al considerar que Argentina es una nación construida por inmigrantes en contraposición con las culturas precolombinas es que el debate toma riqueza. 
La producción cultural en Argentina puede dividirse por subsectores dentro del sector cultural: por una parte, un sector independiente o también llamado alternativo, y por la otra uno empresarial ${ }^{17}$ (Hantouch y Sánchez-Salinas, 2018). Tanto en el ámbito escénico como en el audiovisual esta impronta genera modos de producción diferentes en cada subsector. En este sentido, las políticas culturales tienden a mejorar, o al menos deberían hacerlo, la producción independiente, así como a facilitar la empresarial, al colaborar de manera activa a que la primera tenga apoyos y la segunda facilidades. Por eso, en la actualidad, la producción independiente argentina cuenta con apoyos económicos del Estado a través de líneas de subsidios o becas y la empresarial tiene facilidades, como, por ejemplo, con exenciones impositivas (Algán, 2019). En algunas medidas para el sector audiovisual, también el sector empresarial se ve beneficiado como en el caso del Distrito Audiovisual de la Ciudad de Buenos Aires, el cual promovía que, por pertenecer al distrito, no se les cobraran impuestos al sello e impuestos brutos por diez años, marcando aún más esta distancia entre la capital y el interior.

En el caso de la producción independiente dentro del ámbito escénico el modo es la sociedad accidental de trabajo, mejor conocida como cooperativa de actores, la cual reparte ganancia por puntaje en función de la recaudación. Es la forma convencional más utilizada, aunque su legalidad es dudosa en virtud de no generar aportes jubilatorios, cubrir riesgos de trabajo o despidos. En el caso de la producción empresarial rige desde hace más de cuarenta años el Convenio 307/73, por el cual se estipulan los límites de contratación, los derechos y deberes de los artistas y los empresarios. En el caso del cine, el SICA —el sindicato de trabajadores de cine- estipula las jornadas laborales y los honorarios, de manera que la parte técnica está mejor cubierta. De todas maneras, aprovechando la naturaleza proyectual de la industria en la cual se encuentran, la mayoría de las relaciones se establecen mediante independientes que emiten una factura, de manera que no presentan aportes, obra social y demás beneficios laborales; es decir, están por su cuenta.

\footnotetext{
17 El sector público u oficial también produce contenido, tal como sucede en las artes escénicas o en la televisión. Se excluye de este análisis porque no representa en sí mismo una fuerza productiva con afán de lucro, sino que su objetivo está fijado en el incremento del capital cultural de la ciudadanía. Además, tiene una forma de contratación específica regida por la Ley de Empleo Público Nacional N. ${ }^{\circ} 25.164$ y no es alcanzable por los beneficios que promueven los institutos que se están abordando.
} 
En materia legal, el último cambio que afectó de forma directa este modo de producción fue la sanción de la denominada ley del actor (Ley Nacional 27.203 de 2015), la cual está vigente. Lamentablemente, el decreto que la reglamentó se encuentra suspendido y a la espera de un fallo de la Corte Suprema de Justicia, debido a que el sindicato de actores interpuso una demanda por considerar que la reglamentación atenta contra el espíritu de la ley. La norma excede la actividad teatral, alcanza la actividad audiovisual y la publicidad. Esta ley, en caso de destrabarse la cuestión en la vía judicial, puede ser un cimbronazo para la producción cultural en todas las esferas porque supone un incremento en los aportes del talento artístico que impactará en la estructura de costos de los emprendimientos culturales. Como se sabe, los empresarios no podrán trasladar esa suma al precio de la entrada con lo cual habrá que ver si esto supondrá una merma en la producción o una baja en los estándares de calidad.

En cuanto a los actores en la producción audiovisual, aquí entra en juego también el factor de la repetición por el uso de imagen. Consultado por este tema, Ramiro San Honorio, actual presidente del Consejo de Nuevos Medios en Argentores ${ }^{18}$, se expresó y le dio la importancia del derecho por la imagen y de cuántas veces los actores quedaban por fuera del negocio. Un ejemplo claro de ello son las $\mathrm{PNT}^{19}$, en las que el medio cierra un contrato para mostrar determinado producto y el actor es quien lleva adelante la acción, pero sin percibir ingresos. Sin mencionar que, si las repeticiones no estuvieran regladas, un contenido audiovisual que se repita mucho tiempo en pantalla perjudica al actor en cuanto a que, como el público percibe que está en pantalla, no lo llaman para desarrollar otro personaje.

Ambas industrias en Argentina cuentan con larga tradición. Muchas de estas tradiciones son malos usos. Por una parte, el apoyo del Estado y los institutos al sector independiente y cómo el sector empresarial encuentra su punto de equilibrio en otro tipo de público y contenido. Así, el productor mediano es el que queda desprotegido y se deja una polarización que, en el caso del ámbito

\footnotetext{
18 Argentores se define a sí misma como «una asociación civil de carácter profesional y mutual. Fue fundada el 11 de septiembre de 1910 inicialmente con el nombre de Sociedad Argentina de Autores Dramáticos, y posteriormente instituida, a fines de 1934, con la designación actual» (Argentores, s. f.).

19 La sigla significa publicidad no tradicional. Se refiere a que en la narrativa un actor menciona una marca o desarrolla una acción mostrando el producto.
} 
audiovisual, ya que la producción independiente tiene exhibición muy limitada y no busca llegar a un gran público, agranda la distancia entre difusión y producción. También, dentro de lo empresarial, se cuenta con productoras de contenido televisivo que, a fin de promocionar a sus artistas, producen filmes apoyados en este star system televisivo, así como las cadenas de TV también se suman como socios en la comunicación y distribución de las películas (Getino, 2005).

De esta forma, se detectan dos grandes áreas de vacancia: por una parte, la formación de un espectador y de públicos que estén más cerca de quienes cuentan las historias y pidan aquellas que quieren escuchar. Por otra, en el caso del audiovisual, se da por un marco empresarial que está reflejado en lo legal: dado que las distribuidoras, en su mayor medida, se dedican a los filmes extranjeros o son parte de medios extranjeros, el apoyo del instituto es prácticamente inexistente para taquilla y distribución. ¿Cómo aprenderían los productores locales a conectarse y comunicarse con el público? Así, el éxito de un largometraje argentino está muchas veces ligado a su desempeño en mercados internacionales. No es un dato menor que las OTT no revelen datos ni números de consumo y audiencias, lo que ayudaría también a acercar la película al público. Manuel García, presidente de la Cámara Argentina de Distribuidores Independientes de Cine, explica que esos datos de tendencias y comportamientos permitirían comprender dónde está el público que está interesado en consumir determinada historia audiovisual, y las OTT, al no presentar un alto retorno de la inversión y unos algoritmos tampoco explicados de recomendación, terminan por ser, en sus palabras, «cementerios de películas» (M. García, comunicación personal, 2020). 


\section{Reflexiones finales}

Se ha problematizado la historia argentina a través de dos institutos nacionales que son claves para vehiculizar las políticas culturales. Si bien en los derechos culturales ya se prescribe el acceso a la cultura y el derecho a ser parte de las manifestaciones culturales, no debe dejarse de lado que las políticas son un puente para reflexionar el lugar que se le da a la cultura en determinado contexto. Por esta razón, la relación entre cultura, identidad, política y sociedad ha sido el terreno de reflexiones de este texto. Tanto las leyes vinculadas al teatro como las vinculadas al cine en Argentina son leyes de protección de la actividad cultural, reconociendo las amenazas a las que se ve expuesta.

En el caso del cine, sin el instituto no sería posible una producción estable a causa de la competencia internacional. Sin estas medidas proteccionistas y de apoyo a una industria local no habría garantías de que tuvieran siquiera las producciones una fecha de estreno. El marco que establece la ley de cine empareja la presencia en cartelera, pero el hecho de no incluir distribuidores y exhibidores, de modo que se permite así la entrada de capitales extranjeros para dominar las áreas de contacto con los públicos, hace que siempre se sienta un contenido de resistencia, de intentar hacer frente a los tanques cinematográficos extranjeros. En el caso del teatro las acciones de su instituto están más bien orientadas a la manutención de salas y a la sustentación de las producciones independientes. El público argentino desconoce su cine los informes sobre consumos culturales, discontinuos y parciales, dan cuenta de ello-. En el caso del teatro todavía se convive con una suerte de resabio de star system donde el proceso de selección de compra de los consumidores culturales está mediado por la presencia de una figura o de un director aclamado antes que por la calidad de la obra teatral.

En este sentido, los Estados, por medio de los gobiernos, deben tener una postura de largo plazo frente a esta situación con el objeto de apuntalar e incentivar el efecto multiplicador del sector cultural en las economías que le son conexas (Arriaga-Navarrete y González-Pérez, 2016). En el caso del 
audiovisual, casi que parece un fantasma recurrente la vinculación con el turismo. Potenciar una locación hace no solo que se deje dinero en el lugar durante el rodaje, sino que sea un argumento de venta para que otros vengan a visitarlo. Es renovar la mirada sobre un destino que se conoce previamente, para lo cual también es necesario defender los espacios: es momento también de preguntarse por el impacto social y ambiental de los grandes rodajes en determinados lugares (Getino, 2005). No solo es el caso este de los espacios naturales o históricos, sino también de los que constantemente son sets afectando circulación y cuidados como es el caso del barrio de San Telmo. ¿Cuál es el costo de que ese patrimonio se pierda del desgaste entre el turismo, los rodajes, cortar las calles y la circulación permanente? ¿Bajo la palabra de la cultura, se reemplaza patrimonio cultural? ¿Vale más la mirada de la maravillosa película de Wong Kar Wai sobre el barrio de La Boca ${ }^{20}$ que Caminito en sí?

En un plano empresarial, como ya se ha mencionado, la concentración en Capital Federal trae, casi por nostalgia, la vieja discusión de unitarios versus federales. Ese foco ya no es cultural en cuanto a que el paradigma por el cual se lo analiza en la actualidad no es de una única identidad cultural nacional, sino desde la diversidad. Se habla hoy en día de esa rivalidad en el sentido de repartición de bienes económicos, valores simbólicos y culturales. Si la producción se concentra y desarrolla en un solo foco del país, a espaldas del resto del territorio, la polarización es cada vez mayor. Aquí es que está la nostalgia: Buenos Aires es el único puerto y aeropuerto internacional que funciona todo el año, lo que hace cada vez más dependientes a otras ciudades de la Capital Federal.

Los institutos intentan llevar programas de formación ${ }^{21}$ y distribución a distintos espacios ${ }^{22}$ para movilizar focos de producción. En ambos casos, el ámbito empresarial no está listo porque no son solo los aeropuertos y puertos a los que les falta estructura: los teatros, los escenarios y las salas no cuentan con la misma tecnología que tienen en la Capital Federal. En el caso

\footnotetext{
20 En referencia a Happy Together de 1997, ganadora a Mejor Directory Actor en el Festival de Cannes de ese año.

21 El INCAA cuenta con un instituto de formación de directores y guionistas que abrió nuevas sedes fuera de Buenos Aires durante la gestión de Pablo Rovito.

22 Ejemplo de esto es el Festival Internacional de Cine de las Alturas, en el cual se acerca la cartelera comercial e independiente nacional y regional a los espectadores de Jujuy, junto con charlas y talleres abiertos y gratuitos.
} 
del teatro, el caso se complejiza cuando la obra que debe montarse necesita otra estructura que no sea una disposición a la italiana. Las giras, así, son de obras que han tenido buena repercusión en Buenos Aires y luego se llevan a algunos otros centros urbanos. El flujo cultural parece siempre venir desde allí y no generarse internamente. Cuando se da a la inversa, están vinculados con aspectos turísticos: conocer una locación y hacer planes turísticos propuestos en el momento de festivales o espectáculos que se dan en el marco de un festejo regional.

Las industrias culturales se imponen sobre la lógica del arte escénico que es más efímero y artesanal con relación al audiovisual. Sobre esto —escalabilidad, posibilidades económicas y retorno de la inversión con proyección internacional- es que se basa la diferencia de años en establecerse la ley -una 1994 y la otra 1997-y el control sobre el contenido que ya ha sido mencionado. El cine nace en un contexto de medios de comunicación de masa, en el que el alcance de los mensajes parecía infinito: que al resto del mundo llegara un mensaje no cuidado ha sido un elemento recurrente en más de un país de nuestra querida América Latina. A medida que el streaming penetró en los usos de la sociedad, cada vez más se va transformando el audiovisual: desde sus costos, pasando por su cadena de valor, hasta los planos utilizados — ¿será la muerte del plano general?, ¿quién aguantaría demasiados planos que no sean a corta distancia en una pantalla de un dispositivo móvil? - . Ahí es que el teatro y su aspecto artesanal resiste: cada vez más lo que no puede reemplazarse es lo experiencial y los intentos audiovisuales han sido torpes con esto: 3D, 4D, D-motion y otros estímulos más. La narrativa está cambiando.

Los institutos responden a la gestión de una política gubernamental y los empresarios a la gestión de una política económica; espectadores desterritorializados, hibridaciones de culturas y no una definición de qué entendemos por cultura; en fin, una cercanía esquiva con los públicos y una poca comunicación con ellos en una era de la economía de atención. Una frase que se le atribuye a Octavio Getino expresa que una nación sin cine es como una casa sin espejos. No nos olvidemos de nosotros mismos. 


\section{Referencias}

Alabarces, P. (1998). Lo que el estado no da, el fútbol no lo presta: los discursos nacionalistas deportivos en contextos de exclusión social. Trabajo presentado en el Encuentro da la Latin American Studies Association, Chicago, EE. UU., 24-26 de septiembre.

Algán, R. S. (2019). Mercado teatral y cadena de valor. Caseros: RGC Libros.

Appadurai, A. (2001). La modernidad desbordada. Buenos Aires: Trilce-FCE.

Argentores (s. f.). Perfil de la organización. Recuperado de https://bit.ly/3cxlloY

Arriaga-Navarrete, R.; González-Pérez, C. R. (2016). Efectos económicos del sector cultural en México, Análisis Económico, XXXI(77), 219-246.

Arlt, R. (1999). Crónicas. Buenos Aires: El Aleph Ediciones.

Batlle, D. (27 de diciembre de 2018). En 2018 volvió a bajar la cantidad de público en los cines, aunque hubo algunas gratas sorpresas. La Nación. Recuperado de https://bit.ly/32VZn22

Bauman, Z. (2013). La cultura en el mundo de la modernidad líquida. Buenos Aires: Fondo de Cultura Económica.

Bhabha, H. (1994) El lugar de la cultura. Buenos Aires: Editorial Manantial.

Bayardo, R. (2019). Algunas coordenadas de la gestión cultural en la Argentina. En E. Fuentes-Firmani y J. A. Tasat (Coord.) Gestión cultural en la Argentina (13-32). Caseros: RGC Libros.

Bonet, LL. (2007). El lugar de la economía de la cultura como disciplina contemporánea. En Observatorio Cultural (Comp.) Economía de la cultura. (17-34). Buenos Aires: Facultad de Ciencias Económicas de la Universidad de Buenos Aires, Posgrado en Administración de las Artes del Espectáculo.

Cinelli, J. P. (13 de febrero de 2020). Las razones del cierre del Gaumont. Página 12. Recuperado de https://bit.ly/304p7aS

Constitución de la Nación Argentina. (1999). Buenos Aires: Kapelusz.

Delupi, J.; Vibes, F. (2006). Derecho del entretenimiento. Buenos Aires: Ad-hoc.

Dubatti, J. (2009). Concepciones de teatro: poéticas teatrales y bases epistemológicas. Buenos Aires: Colihue. 
Dubatti, J. (2012). Cien años de teatro argentino. Buenos Aires: Biblos.

El teatro cumple el sueño del instituto propio. (30 de septiembre de 1997). La Nación. Recuperado de https://bit.ly/2FUC3ZZ

El Instituto Nacional del Teatro, en marcha. (7 de abril de 1998). La Nación. Recuperado de https://bit.ly/3038k84

Fuentes-Firmani, E. (2019). ¿Y eso con qué se come? Reflexiones sobre la gestión cultural en la Argentina. En E. Fuentes; J. Tasat (Coords.) Gestión cultural en la Argentina. (43-61). Caseros: RGC Libros.

García, S. (3 de agosto de 2018). Cine durante la dictadura. Leer cine. Recuperado de https://bit.ly/2G6FKvb

García-Canclini, N. (1983). Las políticas culturales en América Latina. Chasqui. Revista Latinoamericana de Comunicación, (7), 18-26.

García-Canclini, N. (2005). Definiciones en transición. En D. Mato (Comp.) Cultura, política y sociedad. Perspectivas latinoamericanas. (69-81). Buenos Aires: Consejo Latinoamericano de Ciencias Sociales (Clacso).

Getino, O. (2005). Cine argentino, entre lo posible y lo deseable. Buenos Aires: Ciccus.

Getino, O. (2007). El peso de lo intangible. En Observatorio Cultural (Comp.) Economía de la cultura. (17-34). Buenos Aires: Facultad de Ciencias Económicas de la Universidad de Buenos Aires, Posgrado en Administración de las Artes del Espectáculo.

Grimson, A. (2011). Los límites de la cultura. Buenos Aires: Siglo Veintiuno.

Hall, S.; Du Gray, P. (Comp.) (1996) Cuestiones de identidad cultural. Buenos Aires: Amorrortu Editores.

Hantouch, J.; Sánchez-Salinas, R. (2018). Cultura independiente: cartografía de un sector movilizado en Buenos Aires. Caseros: RGC Libros.

Karush, M. (2013). Cultura de clase. Radio y cine en la creación de una Argentina dividida (1920-1946). Buenos Aires: Ediciones Ariel.

Ley Nacional N. 24.377 (28 de septiembre de 1994). Boletín Oficial núm. 27.999, octubre 19 de 1994.

Ley Nacional N.² 24.800 (28 de abril de 1997). Boletín Oficial núm. 28.628, mayo 19 de 1997. 
Ley Nacional N. ${ }^{\circ} 27.203$ (28 de octubre de 2015). Boletín Oficial núm. 33.265, noviembre 26 de 2015.

Luna, F. (1997). Breve historia de los argentinos. Buenos Aires: Planeta.

Mendes-Calado, P. (2019). Política cultural. Crítica y utopía. En E. Fuentes; J. Tasat (Coords.) Gestión cultural en la Argentina. (287-300). Caseros: RGC Libros.

Ministerio de Cultura. (s. f.). Con 217 estrenos, 2017 tuvo récord de películas argentinas. Recuperado de https://bit.ly/2FZ9TwC

Pellarolo, S. (1997). Sainete criollo: democracia y representación: el caso de Nemesio Trejo. Buenos Aires: Corregidor.

Peña, F. (2012). Cien años de cine argentino. Buenos Aires: Biblos.

Olmos, H.; Santillán-Güemes, R. (Comp.) (2004) El gestor cultural. Ideas y experiencias para su capacitación. Buenos Aires: Ediciones Ciccus.

Sarlo, B. (1988). Una modernidad periférica. Buenos Aires 1920 y 1930. Buenos Aires: Nueva Visión.

Schoo, E.; Sirven, P. (2010). Breve historia del espectáculo en la Argentina. Buenos Aires: El Ateneo.

Secretaría de Cultura. (24 de octubre de 2019). Ficer: turismo cinematográfico y la potencia de las locaciones entrerrianas. Entrerios.gov.ar. Recuperado de https:// bit.ly/3mWSJRo

Tesson, C. (2012). Teatro y cine. Madrid: Paidós.

Throsby, D. (2001). Economía y cultura. Madrid: Cambridge University Press.

Unesco. (12 de noviembre de 1966). Declaración de los Principios de la Cooperación Cultural Internacional. Recuperado de https://bit.ly/3cxJbre

Williams, R. (1988) Marxismo y literatura. Barcelona: Península.

Wortman, A. (2005). El desafío de las políticas culturales en la Argentina. En D. Mato (Comp.) Cultura, política y sociedad. Perspectivas latinoamericanas. Buenos Aires: Clacso. Recuperado de https://bit.ly/32WSqOj 